\title{
On Poincaré Series of Unicritical Polynomials at the Critical Point
}

\author{
Juan Rivera-Letelier • Weixiao Shen
}

Received: 14 January 2013 / Accepted: 22 January 2013 / Published online: 19 March 2013

(C) School of Mathematical Sciences, University of Science and Technology of China and Springer-Verlag Berlin Heidelberg 2013

\begin{abstract}
In this paper, we show that for a unicritical polynomial having a priori bounds, the unique conformal measure of minimal exponent has no atom at the critical point. For a conformal measure of higher exponent, we give a necessary and sufficient condition for the critical point to be an atom, in terms of the growth rate of the derivatives at the critical value.
\end{abstract}

Keywords Complex dynamics · Julia sets · Poincaré series · Summability condition Mathematics Subject Classification (2010) 37F35

\section{Introduction}

Let $f: \mathbb{C} \rightarrow \mathbb{C}$ be a polynomial of degree at least 2 , and let $J(f)$ denote its Julia set. An important tool to study the fractal dimensions of the Julia set is the PattersonSullivan conformal measures. Given $t>0$, a conformal measure of $f$ of exponent $t$ is a Borel probability measure $\mu$ supported on $J(f)$, such that for each Borel set $E$ on which $f$ is injective we have

$$
\mu(f(E))=\int_{E}|D f|^{t} d \mu .
$$

J. R.-L. was partially supported by FONDECYT grant 1100922, Chile.

W. S. was partially supported by National University of Singapore grant C-146-000-032-001.

J. Rivera-Letelier

Facultad de Matemáticas, Pontificia Universidad Católica de Chile, Avenida Vicuña Mackenna 4860,

Santiago, Chile

e-mail: riveraletelier@mat.puc.cl

W. Shen $(\bowtie)$

Block S17, 10 Lower Kent Ridge Road, Singapore 119076, Singapore

e-mail: matsw@nus.edu.sg 
Sullivan showed that for every polynomial there is $t>0$ and a conformal measure of $f$ of exponent $t$ [25]. Moreover, Denker, Przytycki, and Urbański showed that the minimal exponent for which such a measure exists coincides with the hyperbolic dimension of $f$, defined by

$$
\operatorname{HD}_{\text {hyp }}(f):=\sup \{\operatorname{HD}(X): X \text { hyperbolic set of } f\},
$$

where $\operatorname{HD}(X)$ denotes the Hausdorff dimension of $X$ (see $[2,14,20,22,26])$. In the uniformly hyperbolic case, there is a unique conformal measure of minimal exponent, and this measure coincides with the normalized Hausdorff measure of dimension equal to $\operatorname{HD}(J(f))$. Under certain non-uniform hyperbolicity assumptions, it has also been proved that there is a unique conformal measure of minimal exponent, and that this measure is supported on the "conical Julia set", which roughly speaking is the expanding part of the Julia set (see $[3,17,21,24,26])$ and references therein. In these cases, the conformal measure of minimal exponent is used to prove that the hyperbolic dimension coincides with the Hausdorff and the box dimensions of the Julia set. However, there are conformal measures of minimal exponent that are not supported on the conical Julia set [26].

In this paper, we shall study atoms of conformal measures of polynomials having precisely 1 critical point; we call such a polynomial unicritical. Note that if $f$ is a unicritical polynomial, then its degree $d$ is at least 2, and there is $c$ in $\mathbb{C}$ such that $f$ is affine conjugate to the polynomial $z^{d}+c$. A unicritical polynomial written in this form is normalized. We shall make the following assumption.

Definition 1 Let $f$ be a unicritical polynomial whose critical point is non-periodic and recurrent. Assume for simplicity that $f$ is normalized, so its critical point is 0 . Then we say $f$ has a priori bounds, if there exists $\tau>0$ such that for each $\varepsilon>0$ there exists a topological disk $V$ containing 0 , satisfying $\operatorname{diam}(V)<\varepsilon$, and such that the following holds: For each integer $n \geq 1$ such that $f^{n}(0)$ is in $V$, the connected component $U$ of $f^{-n}(V)$ that contains 0 satisfies $\bar{U} \subset V$, and there is annulus $A$ contained in $V \backslash \bar{U}$, enclosing $U$, and whose modulus is at least $\tau$.

Examples of unicritical polynomials having a priori bounds include at most finitely renormalizable polynomials without neutral cycles [4, 6, 7], all real polynomials [10, $11]$, and some infinitely renormalizable complex polynomials [5, 12]. Recall that for an integer $s \geq 1$, a unicritical polynomial $f$ of degree $d \geq 2$ is renormalizable of period $s$, if there are Jordan disks $U$ and $V$ such that $\bar{U}$ is contained in $V$, and such that the following properties hold:

- $f^{s}: U \rightarrow V$ is $d$-to- 1 ;

- $U$ contains the critical point of $f$, and for each $j$ in $\{1, \ldots, s-1\}$ the set $f^{j}(U)$ does not contain it;

- The set $\left\{z \in U: f^{s n}(z) \in U, n=0,1, \ldots\right\}$ is a connected proper subset of $J(f)$.

Moreover, $f$ is infinitely renormalizable, if there are infinitely many integers $s$ such that $f$ is renormalizable of period $s$.

In the statement of the following theorem we use the fact that for a unicritical polynomial $f$ having a priori bounds there is a unique conformal measure of exponent $t=\operatorname{HD}_{\text {hyp }}(f)$ for $f$ (see [16, Theorem 1]). 
Theorem A Let $f$ be a unicritical polynomial having a priori bounds. Then the conformal measure $\mu$ of minimal exponent of $f$ does not have an atom at the critical point of $f$.

We remark that in this result, it is essential that we consider the conformal measure of minimal exponent, as opposed to a conformal measure of higher exponent. In fact, every unicritical map $f$ that is not uniformly hyperbolic has, for each $t>\operatorname{HD}_{\text {hyp }}(f)$, a conformal measure of exponent $t$ (see [3]) for the case $f$ satisfies the ColletEckmann condition, and $[18,19]$ for the case $f$ does not. In many cases, even if $f$ has a priori bounds, for each $t>\operatorname{HD}_{\text {hyp }}(f)$ there is conformal measure of exponent $t$ that is supported on the backward orbit of the critical point.

For a unicritical polynomial $f$, the existence of an atom at a point $w$ for a conformal measure of $f$ of exponent $t$, is closely related to convergence of the following series:

$$
\mathcal{P}(w, t):=\sum_{n=0}^{\infty} \sum_{z \in f^{-n}(w)}\left|D f^{n}(z)\right|^{-t} ;
$$

it is the Poincaré series at $w$ of exponent $t$. In fact:

- If a conformal measure of exponent $t$ has an atom at $w$, then $\mathcal{P}(w, t)<\infty$;

- A conformal measure of exponent $t$ has an atom at the critical point $c_{0}$ if and only if $\mathcal{P}\left(c_{0}, t\right)<\infty$.

Theorem B Let $f$ be a unicritical polynomial having a priori bounds, and let $t>$ $\operatorname{HD}_{\text {hyp }}(f)$ be given. Assume $f$ is normalized so its critical point is 0 . Then the series $\mathcal{P}(0, t)$ is finite if and only if $\sum_{n=0}^{\infty}\left|D f^{n}(f(0))\right|^{-t / d}$ is finite.

\subsection{Strategy and Organization}

We derive Theorem A from Theorem B arguing by contradiction. If the conformal measure of minimal exponent had an atom at the critical point, by Theorem B the derivatives at the critical value would grow to infinity. Together with the a priori bounds hypothesis, this implies that the map is "backward contracting" (Theorem 1), so the results of [24] apply to this map; in particular, the Poincaré series at the critical point diverges. This contradicts the existence of an atom at the critical point. In Sect. 2 we fix some notation and terminology and recall the definition of backward contracting maps. We deduce Theorem A from Theorem B in Sect. 3, after proving Theorem 1.

To prove Theorem B, we show that in either case the map is backward contracting Theorem 1(1). This allows us to use the characterization for backward contracting maps of the summability condition given in [9]. We recall this result in Sect. 3, as Theorem 1(2). To prove the direct implication in Theorem B, we divide the integral of the backward contraction function, characterizing the summability condition, into integrals over intervals bounded by consecutive close return scales. Then we show that each of these integrals is bounded by one of the terms in the Poincaré series up to a multiplicative constant (Lemma 5). This is done in Sect. 4. The proof of the reverse 
implication in Theorem B occupies Sect. 5 and is more involved. We use a discretized sequence of scales to code each iterated preimage of the critical point. To do this, we consider the largest scale whose pull-back is conformal, and consider the "critical hits" when pulling back the previous scale, as a code. One of the crucial estimates is the contribution in the Poincaré series of those iterated preimages of the critical point for which a certain ball can be pulled back conformally (Lemma 10). This estimate is done using one of the main results of [24]. For a backward contracting map the diameter of pull-backs decreases at least at a super-polynomial rate.

\section{Preliminaries}

We endow $\mathbb{C}$ with its norm distance, and for a bounded subset $W$ of $\mathbb{C}$ we denote by $\operatorname{diam}(W)$ the diameter of $W$. Moreover, for $\delta>0$ and for a point $z$ of $\mathbb{C}$, we denote by $B(z, \delta)$ the ball of $\mathbb{C}$ centered at $z$ and of radius $\delta$.

A topological disk is an open subset of $\mathbb{C}$ homeomorphic to the unit disk, and that is not equal to $\mathbb{C}$. We endow such a set with its hyperbolic metric. If $V$ and $V^{\prime}$ are topological disks such that $\overline{V^{\prime}} \subset V$, we denote by $\bmod \left(V ; V^{\prime}\right)$ the supremum of the moduli of all annuli contained in $V \backslash V^{\prime}$ that enclose $V^{\prime}$ (see $[1,15]$ for background).

Throughout the rest of this paper we fix an integer $d \geq 2$, a parameter $c$ in $\mathbb{C}$, and

put $f(z):=z^{d}+c$. Moreover, we assume 0 is non-periodic and recurrent by $f$; this implies that 0 is in the Julia set of $J(f)$. Given a subset $V$ of $\mathbb{C}$, and an integer $n \geq 0$, each connected component of $f^{-n}(V)$ is called a pull-back of $V$ by $f^{n}$. When $n \geq 1$, a pull-back of $V$ is critical if it contains 0 .

\subsection{Backward Contraction}

In this section we recall the definition of "backward contraction" from [23], and compare it with a variant from [9].

For each $\delta>0$, put $\widetilde{B}(\delta):=B\left(0, \delta^{1 / d}\right)$, and

$$
r(\delta):=\sup \left\{r>0: \begin{array}{l}
\text { for every pull-back } W \text { of } \widetilde{B}(\delta r), \\
\operatorname{dist}(W, c) \leq \delta \text { implies } \operatorname{diam}(W)<\delta
\end{array}\right\} .
$$

Given $r_{0}>1$, the map $f$ is backward contracting with constant $r_{0}$, if for every sufficiently small $\delta$ we have $r(\delta)>r_{0}$. Moreover, $f$ is backward contracting if $r(\delta) \rightarrow \infty$ as $\delta \rightarrow 0$.

For each $\delta>0$, put

$$
R(\delta):=\inf \left\{\frac{\delta}{\operatorname{diam}(U)}: U \text { pull-back of } \widetilde{B}(\delta) \text { containing } c\right\} .
$$

Clearly, for every $\delta>0$ and $\delta^{\prime}>\delta$ we have $R(\delta) \geq\left(\delta / \delta^{\prime}\right) R\left(\delta^{\prime}\right)$. Moreover, for every $\delta>0$ and every $r$ in $(0, r(\delta))$, we have $R(\delta r) \geq r$. So, if $f$ is backward contracting with some constant $r_{0}>1$, then for every sufficiently small $\delta$ we have $R(\delta) \geq r_{0}$. In particular, if $f$ is backward contracting, then $R(\delta) \rightarrow \infty$ as $\delta \rightarrow 0$. 
Lemma 1 There is a constant $\rho_{0}>1$ independent of $f$ and $d$, such that for every $\delta>0$ satisfying $R(\delta) \geq \rho_{0}^{d}$, and every $\delta^{\prime}$ in $[\delta / R(\delta), \delta)$, we have $r\left(\delta^{\prime}\right) \geq \rho_{0}^{-d} \delta / \delta^{\prime}$. In particular, if there is $R_{0} \geq \rho_{0}^{d}$ such that for every sufficiently small $\delta>0$ we have $R(\delta) \geq R_{0}$, then $f$ is backward contracting with constant $\rho_{0}^{-d} R_{0}$. Moreover, $f$ is backward contracting if and only if $R(\delta) \rightarrow \infty$ as $\delta \rightarrow \infty$.

Proof Let $\rho_{0}>1$ be sufficiently large so that for every pair of topological disks $U$ and $V$ such that

$$
\bar{U} \subset V \quad \text { and } \bmod (V ; U) \geq \log \rho_{0},
$$

we have $\operatorname{diam}(U)<\operatorname{dist}(U, \partial V)$.

Fix $\delta>0$ such that $R(\delta) \geq \rho_{0}^{d}$, and $\delta^{\prime}$ in $[\delta / R(\delta), \delta)$. To prove the lemma it suffices to show that for every integer $n \geq 0$, and every pull-back $W$ of $\widetilde{B}\left(\rho_{0}^{-d} \delta\right)$ by $f^{n}$ satisfying $\operatorname{dist}(W, c) \leq \delta^{\prime}$, we have $\operatorname{diam}(W)<\delta^{\prime}$. We proceed by induction in $n$. When $n=0$, we have $W=\widetilde{B}\left(\rho_{0}^{-d} \delta\right)$. If $c$ is in $\widetilde{B}(\delta)$, then by the definition of $R(\delta)$ we have

$$
\operatorname{diam}(W)<\operatorname{diam}(\widetilde{B}(\delta)) \leq \delta / R(\delta) \leq \delta^{\prime} .
$$

Otherwise, by the definition of $\rho_{0}$ we have

$$
\operatorname{diam}(W)<\operatorname{dist}(W, \partial \widetilde{B}(\delta)) \leq \operatorname{dist}(W, c) \leq \delta^{\prime} .
$$

This proves the desired assertion when $n=0$. Let $n \geq 1$ be an integer such that the desired assertion holds with $n$ replaced by each element of $\{0, \ldots, n-1\}$. Let $W$ be a pull-back of $\widetilde{B}\left(\rho_{0}^{-d} \delta\right)$ by $f^{n}$ satisfying $\operatorname{dist}(W, c) \leq \delta^{\prime}$. If the pull-back $\widehat{W}$ of $\widetilde{B}(\delta)$ containing $W$ contains $c$, then by the definition of $R(\delta)$ we have

$$
\operatorname{diam}(W)<\operatorname{diam}(\widehat{W}) \leq \delta / R(\delta) \leq \delta^{\prime} .
$$

So we suppose $\widehat{W}$ does not contain $c$. If $f^{n}$ is conformal on $\widehat{W}$, then

$$
\bmod (\widehat{W} ; W)=\bmod \left(\widetilde{B}(\delta) ; \widetilde{B}\left(\rho_{0}^{-d} \delta\right)\right)=\log \rho_{0} .
$$

So by the definition of $\rho_{0}$ we have

$$
\operatorname{diam}(W)<\operatorname{dist}(W, \partial \widehat{W}) \leq \operatorname{dist}(W, c) \leq \delta^{\prime} .
$$

It remains to consider the case where $f^{n}$ is not conformal on $\widehat{W}$, so there is $m$ in $\{0, \ldots, n-1\}$ such that $f^{m}(\widehat{W})$ contains 0 . Then $f^{m+1}(\widehat{W})$ contains $c$, and by definition of $R(\delta)$ we have

$$
\operatorname{diam}\left(f^{m+1}(\widehat{W})\right) \leq \delta / R(\delta) \leq \rho_{0}^{-d} \delta .
$$

This implies that $f^{m+1}(\widehat{W})$ is contained in $B\left(c, \rho_{0}^{-d} \delta\right)$, and therefore that $f^{m}(\widehat{W})$ is contained in $\widetilde{B}\left(\rho_{0}^{-d} \delta\right)$. Using the induction hypothesis, we conclude that $\operatorname{diam}(W)<\delta^{\prime}$. This completes the proof of the induction step and of the lemma. 


\subsection{Nice Sets and Children}

The purpose of this section is to prove a general lemma about backward contracting maps that is used in Sect. 5.1 to prove Theorem B.

A topological disk $V$ containing 0 is a nice set for $f$ if for every integer $n \geq 1$ the set $f^{n}(\partial V)$ is disjoint from $V$. Given $\lambda>0$, a nice set $V$ for $f$ is $\lambda$-nice, if for every pull-back $U$ of $V$ contained in $V$ we have

$$
\bar{U} \subset V \quad \text { and } \bmod (V ; U) \geq \lambda .
$$

For an integer $m \geq 1$, and a connected open set $V$, a pull-back $W$ of $V$ by $f^{m}$ containing 0 is a child of $V$, if $f^{m}$ maps $W$ onto $V$ as a $d$-to- 1 map.

The following lemma is a more precise version of [24, Lemma 3.15], with the same proof.

Lemma 2 Suppose $f$ is backward contracting. Then for every $s>0$ there is $\delta_{0}>0$, such that for every $\delta$ in $\left(0, \delta_{0}\right]$ there is a nice set $V$ for $f$ satisfying $\widetilde{B}(\delta / 2) \subset V \subset$ $\widetilde{B}(\delta)$, and such that

$$
\sum_{Y: \text { child of } V} \operatorname{diam}(f(Y))^{s} \leq\left(1-2^{-s}\right)^{-1}\left(R(\delta)^{-1} \delta\right)^{s} .
$$

Proof Let $\lambda>0$ be sufficiently large so that for every pair of topological discs $Y$ and $Y^{\prime}$ satisfying

$$
\overline{Y^{\prime}} \subset Y \text { and } \bmod \left(Y ; Y^{\prime}\right) \geq \lambda,
$$

we have $\operatorname{diam}\left(Y^{\prime}\right) \leq \operatorname{diam}(Y) / 2$, see for example [13, Theorem 2.1]. In view of [24, Lemma 3.13], for every sufficiently small $\delta>0$ there is a $\lambda$-nice set $V$ for $f$ such that $\widetilde{B}(\delta / 2) \subset V \subset \widetilde{B}(\delta)$. We prove that the desired assertion holds for this choice of $V$.

For each integer $k \geq 1$, let $Y_{k}$ be the $k$-th smallest child of $V$ and let $s_{k}$ be the integer such that $f^{s_{k}}\left(Y_{k}\right)=V$. By the backward contracting property, we have $\operatorname{diam}\left(f\left(Y_{1}\right)\right) \leq R(\delta)^{-1} \delta$. Note that for each $k \geq 1$ the set $f^{s_{k}}\left(Y_{k+1}\right)$ is contained in a return domain of $V$, so $\bmod \left(V ; f^{s_{k}}\left(Y_{k+1}\right)\right) \geq \lambda$. By the definition of child, the map $f^{s_{k}-1}: f\left(Y_{k}\right) \rightarrow V$ is conformal, thus $\bmod \left(f\left(Y_{k}\right) ; f\left(Y_{k+1}\right)\right)=$ $\bmod \left(V ; f^{s_{k}}\left(Y_{k+1}\right)\right) \geq \lambda$ and therefore $\operatorname{diam}\left(f\left(Y_{k+1}\right)\right) \leq \operatorname{diam}\left(f\left(Y_{k}\right)\right) / 2$. The conclusion of the lemma follows.

\section{A Priori Bounds and Backward Contraction}

In this section we derive Theorem A from Theorem B. To do so, we first establish a sufficient criterion for a unicritical map having a priori bounds to be backward contracting (Theorem 1 below).

In the following theorem we summarize and complement results in $[8,9]$, when restricted to unicritical maps. We state it in a stronger form than what is needed for this section. 
Theorem 1 For each $\tau>0$ there is a constant $\eta>1$, such that if $f$ has a priori bounds with constant $\tau$, then the following properties hold.

(1) Let $R_{0}>1$ be such that for every sufficiently large $n$ we have either

$$
\left|D f^{n}(c)\right| \geq \eta R_{0}, \quad \text { or } \min _{\zeta \in f^{-n}(0)}\left|D f^{n}(\zeta)\right| \geq\left(\eta R_{0}\right)^{1 / d}
$$

Then for every sufficiently small $\delta>0$ we have $R(\delta) \geq R_{0}$.

(2) For each $t>0$, the sum $\sum_{n=0}^{\infty}\left|D f^{n}(c)\right|^{-t}$ is finite if and only if $\int_{0+}^{1} R(\delta)^{-t} \frac{d \delta}{\delta}$ is finite.

When $\left|D f^{n}(c)\right|$ is eventually bounded from below by $\eta R_{0}$, part 1 is given by (the proof of) [8, Theorem A]. Part 2 is a direct consequence of part 1 and [9, Theorems 1.3 and 1.4], together with Lemma 1 and [23, Corollary 8.3].

To prove this theorem, we shall use the following variation of the Koebe distortion theorem.

Lemma 3 For each $\tau_{0}>0$ there is a constant $C_{@}>1$, such that for every pair of topological disks $V$ and $V^{\prime}$ containing 0 , and satisfying

$$
\overline{V^{\prime}} \subset V \quad \text { and } \bmod \left(V ; V^{\prime}\right) \geq \tau_{0}
$$

the following property holds. Let $s \geq 1$ be a integer such that $f^{s}(0)$ is in $V^{\prime}$, and such that $f^{s-1}$ maps a neighborhood $W$ of c conformally onto $V$. Moreover, let $W^{\prime}$ be the pull-back of $V^{\prime}$ by $f^{s-1}$ contained in $W$, and let $\zeta$ in $f^{-s}(0)$ be such that $f(\zeta)$ is in $W^{\prime}$. Then we have

$$
\operatorname{diam}\left(W^{\prime}\right) \leq C_{@} \max \left\{\left|D f^{s}(c)\right|,\left|D f^{s}(\zeta)\right|^{d}\right\}^{-1} \operatorname{diam}\left(f\left(V^{\prime}\right)\right) .
$$

Proof By Koebe distortion theorem there is a constant $K_{1}>1$ that only depends on $\tau_{0}$, such that the distortion of $f^{s-1}$ on $W^{\prime}$ is bounded by $K_{1}$. We thus have,

$$
\begin{aligned}
\operatorname{diam}\left(W^{\prime}\right) & \leq K_{1}\left|D f^{s-1}(c)\right|^{-1} \operatorname{diam}\left(V^{\prime}\right) \\
& =K_{1}\left|D f^{s}(c)\right|^{-1}\left|D f\left(f^{s-1}(c)\right)\right| \operatorname{diam}\left(V^{\prime}\right) .
\end{aligned}
$$

Since $\left|D f\left(f^{s-1}(c)\right)\right|=d\left|f^{s-1}(c)\right|^{d-1} \leq d \operatorname{diam}\left(V^{\prime}\right)^{d-1}$, we obtain

$$
\begin{aligned}
\operatorname{diam}\left(W^{\prime}\right) & \leq d K_{1}\left|D f^{s}(c)\right|^{-1} \operatorname{diam}\left(V^{\prime}\right)^{d} \\
& \leq d 2^{d} K_{1}\left|D f^{s}(c)\right|^{-1} \operatorname{diam}\left(f\left(V^{\prime}\right)\right) .
\end{aligned}
$$

On the other hand, we have $\left|D f^{s-1}(f(\zeta))\right| \leq K_{1}\left|D f^{s-1}(c)\right|$. Using the formula of $f$, we obtain

$$
\left|D f^{s}(\zeta)\right| \leq K_{1}\left(|\zeta| /\left|f^{s-1}(c)\right|\right)^{d-1}\left|D f^{s}(c)\right|
$$


Using Koebe distortion theorem and the formula of $f$ again, we obtain

$$
\left|f^{s-1}(c)\right| \geq K_{2}^{-1}\left|D f^{s-1}(f(\zeta))\right| \cdot|f(\zeta)-c|=\left(d K_{2}\right)^{-1}\left|D f^{s}(\zeta)\right| \cdot|\zeta|,
$$

where $K_{2}>1$ is a constant depending only on $\tau_{0}$. Together with (2), we obtain $\left|D f^{s}(\zeta)\right|^{d} \leq d^{d-1} K^{d}\left|D f^{s}(c)\right|$, where $K=\max \left\{K_{1}, K_{2}\right\}$. Combined with (1), this implies the desired inequality with $C_{@}=(2 d)^{d} K^{d+1}$.

For each topological disk $V$ containing 0 , put

$$
\begin{gathered}
M_{+}(V):=\inf \left\{\left|D f^{n}(c)\right|: n \geq 1, f^{n}(0) \in V\right\}, \\
M_{-}(V):=\inf \left\{\left|D f^{n}(\zeta)\right|^{d}: n \geq 1, \zeta \in V, f^{n}(\zeta)=0\right\},
\end{gathered}
$$

and

$$
M(V):=\max \left\{M_{+}(V), M_{-}(V)\right\} .
$$

Lemma 4 For every constant $\tau_{0}>0$ there is a constant $C_{!}>0$ such that the following property holds. Let $V$ and $V^{\prime}$ be topological disks containing 0 , such that $\overline{V^{\prime}} \subset V, \bmod \left(V ; V^{\prime}\right) \geq \tau_{0}$, and such that every critical pull-back of $V$ is contained in $V^{\prime}$. Then for every critical pull-back $U^{\prime}$ of $V^{\prime}$, we have

$$
\operatorname{diam}\left(f\left(U^{\prime}\right)\right) \leq C_{!} M(V)^{-1} \operatorname{diam}\left(f\left(V^{\prime}\right)\right) .
$$

Proof Let $C_{@}$ be the constant given by Lemma 3. Let $n \geq 1$ be an integer such that $f^{n}(0)$ is in $V^{\prime}$, and let $U$ (resp. $U^{\prime}$ ) be the pull-back of $V$ (resp. $V^{\prime}$ ) by $f^{n}$ containing 0 . It suffices to consider the case that $U$ is not contained in any critical pull-back of $V^{\prime}$. In this case we claim that $f^{n}: U \rightarrow V$ is $d$-to-1. Otherwise, there would exist $m$ in $\{1, \ldots, n-1\}$ such that $f^{m}(U)$ contains 0 . This implies that $f^{m}(U)$ is contained in the pull-back of $V$ by $f^{n-m}$ containing 0 , which is contained in $V^{\prime}$. Thus $f^{m}(U)$ is contained in $V^{\prime}$, and therefore $U$ is contained in the pull-back of $V^{\prime}$ by $f^{m}$ containing 0 . We thus obtain a contradiction that shows that $f^{n}: U \rightarrow V$ is $d$-to-1. Then the lemma follows from Lemma 3 with $C_{!}=C_{@}$.

Given a topological disk $V$, a point $x$ of $V$, and $\Delta>0$, denote by $B_{V}(x, \Delta)$ the ball for the hyperbolic metric of $V$ centered at $x$ and of radius $\Delta$. By Koebe distortion theorem, for every $\Delta>0$ there is $\xi>1$ such that for every topological disk $V$, and every $x$ in $V$, we have

$$
\sup _{z \in \partial B_{V}(x, \Delta)}|z-x| \leq \xi \inf _{z \in \partial B_{V}(x, \Delta)}|z-x| .
$$

Proof of Theorem 1 As mentioned above, part 2 is a direct consequence of part 1, and the combination of [9, Theorems 1.3 and 1.4], Lemma 1, and [23, Corollary 8.3].

To prove part 1 , note that for $\delta>0$ the number $\bmod (\widetilde{B}(\delta) ; \widetilde{B}(\delta / 2))$ is independent of $\delta$; denote it by $\tau_{1}$. On the other hand, let $\tau$ be the constant given by the a priori bounds hypothesis. Note that there is a constant $\Delta>0$ such that if $U$ is a topological 
disk satisfying $\bar{U} \subset V$ and $\bmod (V ; U) \geq \tau$, then the diameter of $U$ with respect to the hyperbolic metric of $V$ is bounded by $\Delta$. Moreover, the number $\bmod \left(V ; B_{V}(0, \Delta)\right)$ is bounded from below by a constant $\tau_{0}>0$ that is independent of $V$. Let $C_{\text {! }}$ be the constant given by Lemma 4 with $\tau_{0}$ replaced by $\min \left\{\tau_{0}, \tau_{1}\right\}$. Let $\xi>1$ be the constant defined above for this choice of $\Delta$, and put $\eta:=4 C_{!} \xi^{d}$.

Let $\varepsilon>0$ be sufficiently small so that for every topological disk $V$ containing 0 and satisfying $\operatorname{diam}(V)<\varepsilon$, we have $M(V) \geq \eta R_{0}$. By the a priori bounds hypothesis, there is such a topological disk so that in addition for every critical pull-back $U$ of $V$ we have $\bar{U} \subset V$ and $\bmod (V ; U) \geq \tau$. By our choice of $\Delta$, this implies that $U$ is contained in $V^{\prime}:=B_{V}(0, \Delta)$. So the hypotheses of Lemma 4 are satisfied for these choices of $V$, and $V^{\prime}$. It follows that for every critical pull-back $U^{\prime}$ of $V^{\prime}$, we have

$$
\operatorname{diam}\left(f\left(U^{\prime}\right)\right) \leq C_{!} M(V)^{-1} \operatorname{diam}\left(f\left(V^{\prime}\right)\right) \leq 4^{-1} \xi^{-d} R_{0}^{-1} \operatorname{diam}\left(f\left(V^{\prime}\right)\right) .
$$

Thus, if we put $\delta_{0}:=\inf _{z \in \partial V^{\prime}}|z|^{d}$, then $\operatorname{diam}\left(f\left(V^{\prime}\right)\right) \leq 2 \xi^{d} \delta_{0}$, and for every critical pull-back $U^{\prime \prime}$ of $\widetilde{B}\left(\delta_{0}\right)$ we have

$$
\operatorname{diam}\left(f\left(U^{\prime \prime}\right)\right) \leq\left(2 R_{0}\right)^{-1} \delta_{0}
$$

This proves $R\left(\delta_{0}\right) \geq 2 R_{0}$.

To complete the proof of part 1 , for each integer $n \geq 1$ put $\delta_{n}:=2^{-n} \delta_{0}$. We prove by induction that for each $n$ we have $R\left(\delta_{n}\right) \geq 2 R_{0}$. The case $n=0$ is shown above. Let $n \geq 1$ be an integer such that $R\left(\delta_{n-1}\right) \geq 2 R_{0}>2$. Then every critical pull-back of $\widetilde{B}\left(\delta_{n-1}\right)$ is contained in $\widetilde{B}\left(\delta_{n}\right)$. So by Lemma 4 , for every critical pull-back $U^{\prime}$ of $\widetilde{B}\left(\delta_{n}\right)$ we have

$$
\operatorname{diam}\left(f\left(U^{\prime}\right)\right) \leq 2 C_{!} M\left(\widetilde{B}\left(\delta_{n-1}\right)\right)^{-1} \delta_{n} \leq\left(2 R_{0}\right)^{-1} \delta_{n} .
$$

This proves $R\left(\delta_{n}\right) \geq 2 R_{0}$ and completes the proof of the induction step. Thus for every $n \geq 0$ we have $R\left(\delta_{n}\right) \geq 2 R_{0}$, and therefore for every $\delta$ in $\left(0, \delta_{0}\right)$ we have $R(\delta) \geq$ $R_{0}$. This completes the proof of part 1 and of the theorem.

Proof of Theorem A assuming Theorem B Let $\mu$ denote the conformal measure of $f$ of minimal exponent $h=\operatorname{HD}_{\text {hyp }}(f)$. Assume by contraction that $\mu$ has an atom at 0 . Then $\mathcal{P}(0, h)<\infty$, hence for each $t>h$ we have $\mathcal{P}(0, t)<\infty$. By Theorem B, it follows that $\sum_{n=0}^{\infty}\left|D f^{n}(c)\right|^{-t / d}<\infty$, hence $\left|D f^{n}(c)\right| \rightarrow \infty$ as $n \rightarrow \infty$. By Lemma 1 and part 1 of Theorem 1, the map $f$ is backward contracting. But then, [24, Proposition 7.3] implies $\mathcal{P}(0, h)=\infty$. We thus obtain a contradiction that completes the proof of the theorem.

\section{Convergence of Poincaré Series Implies Forward Summability}

The purpose of this section is to prove the following proposition, giving one of the implications in Theorem B.

Proposition 1 Suppose $f$ satisfies the a priori bounds condition. Then for each $t>0$ such that $\mathcal{P}(0, t)$ is finite, the sum $\sum_{n=1}^{\infty}\left|D f^{n}(c)\right|^{-t / d}$ is also finite. 
For each $\delta>0$, let $n(\delta)$ be the minimal integer $n \geq 1$ such that $f^{n}(0)$ is in $\widetilde{B}(\delta)$, let $U_{\delta}$ denote the pull-back of $\widetilde{B}(\delta)$ by $f^{n(\delta)}$ that contains 0 , and let $\zeta(\delta)$ be a point of $f^{-n(\delta)}(0)$ in $U_{\delta}$. Clearly, $n(\delta)$ is non-increasing with $\delta$, left continuous, and we have $n(\delta) \rightarrow \infty$ as $\delta \rightarrow 0$. In view of part 2 of Theorem 1, Proposition 1 is a direct consequence of the following lemma.

Lemma 5 There is $\delta_{0}>0$ such that for each $t>0$ there is $C_{\dagger}>0$ such that the following property holds: For every $\delta_{1}$ and $\delta_{2}$ in $\left(0, \delta_{0}\right)$ satisfying $\delta_{1}<\delta_{2}$, and such that $n(\cdot)$ is constant on $\left(\delta_{1}, \delta_{2}\right]$, we have

$$
\int_{\delta_{1}}^{\delta_{2}} R(\delta)^{-t / d} \frac{d \delta}{\delta} \leq C_{\dagger}\left|D f^{n\left(\delta_{2}\right)}\left(\zeta\left(\delta_{2}\right)\right)\right|^{-t} .
$$

The proof of this lemma is after the following one.

Lemma 6 Assume that $f$ is backward contracting, and for each $\delta>0$ let $W_{\delta}$ be the pull-back of $\widetilde{B}(2 \delta)$ by $f^{n(\delta)-1}$ containing $c$. Then for every sufficiently small $\delta$ we have $R(\delta) \geq \delta / \operatorname{diam}\left(W_{\delta}\right)$.

Proof Let $\delta_{0}>0$ be sufficiently small so that for every $\delta$ in $\left(0, \delta_{0}\right)$ we have $r(\delta)>2$. It suffices to show that for each $\delta$ in $\left(0, \delta_{0}\right)$, and every integer $m \geq 0$ such that $f^{m}(c)$ is in $\widetilde{B}(\delta)$, the pull-back $W$ of $\widetilde{B}(\delta)$ by $f^{m}$ containing $c$ is contained in $W_{\delta}$. Clearly $m \geq n(\delta)-1$, and when $m=n(\delta)-1$ we have $W \subset W_{\delta}$. If $m \geq n(\delta)$, then our hypothesis $r(\delta)>2$ implies that $f^{n(\delta)-1}(W)$ is contained in $\widetilde{B}(2 \delta)$. This shows that $W$ is contained in $W_{\delta}$, as wanted.

Proof of Lemma 5 By Koebe Distortion Theorem there is a constant $K>1$ such that for every $\delta>0$, every integer $n \geq 1$, and every pull-back $W$ of $\widetilde{B}(2 \delta)$ by $f^{n}$ for which the corresponding pull-back of $\widetilde{B}(4 \delta)$ is conformal, the distortion of $f^{n}$ on $W$ is bounded by $K$. Let $\delta_{0}>0$ be such that the conclusion of Lemma 6 holds for every $\delta$ in $\left(0, \delta_{0}\right)$. Reducing $\delta_{0}$ if necessary, assume that for each $\delta$ in $\left(0,4 \delta_{0}\right)$ we have $R(4 \delta) \geq 4$.

To prove the lemma, let $\delta_{1}$ and $\delta_{2}$ in $\left(0, \delta_{0}\right)$ be such that $n(\cdot)$ is constant on $\left(\delta_{1}, \delta_{2}\right]$. Put

$$
n:=n\left(\delta_{2}\right) \quad \text { and } \quad \zeta:=\zeta\left(\delta_{2}\right),
$$

and note that $\left|f^{n-1}(c)\right|=\left|f^{n}(0)\right| \leq \delta_{1}^{1 / d}$. Let $\delta$ in $\left(\delta_{1}, \delta_{2}\right]$, and let $W_{\delta}$ (resp. $\widehat{W}_{\delta}$ ) be the pull-back of $\widetilde{B}(2 \delta)$ (resp. $\widetilde{B}(4 \delta)$ ) by $f^{n-1}$ containing $c$. We claim that $f^{n-1}$ is conformal on $\widehat{W}_{\delta}$. Otherwise, we would have $n \geq 2$, and there would exist $m$ in $\{1, \ldots, n-1\}$ such that $f^{m}\left(\widehat{W}_{\delta}\right)$ contains $c$. However, this implies that

$$
\operatorname{diam}\left(f^{m}\left(\widehat{W}_{\delta}\right)\right) \leq 4 \delta / R(4 \delta) \leq \delta,
$$

and therefore that $f^{m-1}\left(\widehat{W}_{\delta}\right)$, and hence $f^{m-1}(0)$, is contained in $\widetilde{B}(\delta)$; we thus obtain a contradiction with the definition of $n=n(\delta)$ that proves that $f^{n-1}$ is conformal on $\widehat{W}_{\delta}$. 
Using Koebe distortion theorem and the formula of $f$, we have

$$
\begin{gathered}
\operatorname{diam}\left(W_{\delta}\right) \leq 2 K(2 \delta)^{1 / d}\left|D f^{n-1}(f(\zeta))\right|^{-1} \\
=2 d K(2 \delta)^{1 / d}\left|D f^{n}(\zeta)\right|^{-1}|\zeta|^{d-1} \\
|\zeta|^{d}=|f(\zeta)-c| \leq K\left|D f^{n-1}(f(\zeta))\right|^{-1}\left|f^{n-1}(c)\right| \\
\leq K \delta_{1}^{1 / d}\left|D f^{n-1}(f(\zeta))\right|^{-1}=d K \delta_{1}^{1 / d}\left|D f^{n}(\zeta)\right|^{-1}|\zeta|^{d-1}
\end{gathered}
$$

and therefore $|\zeta| \leq d K \delta_{1}^{1 / d}\left|D f^{n}(\zeta)\right|^{-1}$. Hence, letting $C_{\ddagger}:=2^{1+1 / d}(d K)^{d}$, by (3) we have

$$
\operatorname{diam}\left(W_{\delta}\right) \leq C_{\ddagger} \delta_{1}\left(\delta / \delta_{1}\right)^{1 / d}\left|D f^{n}(\zeta)\right|^{-d} .
$$

Together with Lemma 6, this implies

$$
R(\delta) \geq C_{\ddagger}^{-1}\left(\delta / \delta_{1}\right)^{1-1 / d}\left|D f^{n}(\zeta)\right|^{d} .
$$

The desired assertion follows with $C_{\dagger}=C_{\ddagger}^{t / d} \int_{1}^{\infty} \eta^{-1-(t / d)(1-1 / d)} d \eta$.

\section{Forward Summability Implies Backward Summability}

In this section we complete the proof of Theorem B. After some estimates in Sect. 5.1, the proof of Theorem B is given in Sect. 5.2.

\subsection{Thickened Grandchildren}

Throughout this section, assume $f$ is backward contracting, so that $R(\delta) \rightarrow \infty$ as $\delta \rightarrow 0$, see Sect. 2.1. Moreover, fix $t>0$, put $\tau:=2^{-d}$, and let $\delta_{0}>0$ be given by Lemma 2 with $s=t / d$. For every integer $q \geq 0$, let $V_{q}$ be the nice set $V$ given by Lemma 2 with $\delta=\tau^{q} \delta_{0}$, so that

$$
\widetilde{B}\left(\tau^{q} \delta_{0} / 2\right) \subset V_{q} \subset \widetilde{B}\left(\tau^{q} \delta_{0}\right)
$$

Note that for every $q \geq 0$ the set $\overline{V_{q+1}}$ is contained in $V_{q}$ and $\bmod \left(V_{q} ; V_{q+1}\right)$ is bounded from below independently of $q$. Reducing $\delta_{0}$ if necessary, assume that for every $\delta$ in $\left(0, \delta_{0}\right]$ we have $R(\delta)>2 \tau^{-2}$.

For each integer $q \geq 0$, let $\mathcal{N}(q)$ be the set of all integers $n \geq 1$ such that $f^{n}(0)$ is in $V_{q}$, and such that the pull-back $W_{n}(q)$ of $V_{q}$ by $f^{n}$ containing 0 is a child of $V_{q}$. By our choice of $\delta_{0}$, the set $W_{n}(q)$ is contained in $\widetilde{B}\left(\tau^{q+2} \delta_{0} / 2\right)$, and hence in $V_{q+2}$; denote by $p_{n}(q)$ the largest integer $p \geq q+1$, such that $W_{n}(q)$ is contained in $V_{p+1}$. Then we have

$$
\operatorname{diam}\left(f\left(W_{n}(q)\right)\right) \geq \tau^{p_{n}(q)+2} \delta_{0} / 2
$$


Combined with Lemma 2, this implies

$$
\sum_{n \in \mathcal{N}(q)} \tau^{t \cdot p_{n}(q) / d} \leq\left(1-2^{-t / d}\right)^{-1}\left(2 R\left(\tau^{q} \delta_{0}\right)^{-1} \tau^{q-2}\right)^{t / d}
$$

Given an integer $q \geq 0$, let $\mathcal{S}(q)$ be the set of all finite sequences of integers $\left(n_{1}, \ldots, n_{k}\right)$, such that there are integers

$$
p_{0}:=q, p_{1}, \ldots, p_{k},
$$

satisfying the following property: for each $i$ in $\{1, \ldots, k\}$ the integer $n_{i}$ is in $\mathcal{N}\left(p_{i-1}\right)$, and $p_{i}=p_{n_{i}}\left(p_{i-1}\right)$. Note that in this case for each $i$ in $\{1, \ldots, k\}$ we have $p_{i} \geq$ $p_{i-1}+1$. In the situation above, we put $p_{\left(n_{1}, \ldots, n_{k}\right)}(q):=p_{k}$.

Let $q \geq 0$ be an integer. For each $k \geq 1$ and $\mathbf{n}=\left(n_{1}, \ldots, n_{k}\right)$ in $\mathcal{S}(q)$, put

$$
|\mathbf{n}|:=n_{1}+\cdots+n_{k} \quad \text { and } \quad k(\mathbf{n}):=k .
$$

Moreover, let $Z_{\mathbf{n}}(q)$ be the set of all points $z$ of $f^{-|\mathbf{n}|}(0)$ such that $f^{|\mathbf{n}|}$ maps a neighborhood of $z$ conformally onto $V_{q+1}$, and such that in the case $k \geq 2$, for each $i$ in $\{2, \ldots, k\}$ the point $f^{n_{k}+\cdots+n_{i+1}}(z)$ is in the pull-back of $V_{p_{\left(n_{1}, \ldots, n_{i-1}\right)}}$ by $f^{n_{i}}$ containing 0 . Note that $Z_{\mathbf{n}}(q) \leq d^{k(\mathbf{n})}$, and that $Z_{\mathbf{n}}(q)$ is contained in $V_{p_{\mathbf{n}}(q)+1}$.

The purpose of this section is to prove the following.

Proposition 2 Suppose $f$ is backward contracting, and put

$$
C_{\#}:=2^{1+2 t / d} d\left(1-2^{-t / d}\right)^{-1} \tau^{-2 t / d} .
$$

Then, for all $q \geq 0$ sufficiently large we have

$$
\sum_{\mathbf{n} \in \mathcal{S}(q)} \sum_{z \in Z_{\mathbf{n}}(q)}\left|D f^{|\mathbf{n}|}(z)\right|^{-t} \leq C_{\#} R\left(\tau^{q} \delta_{0}\right)^{-t / d} .
$$

The proof of this proposition is given after the following lemma.

Lemma 7 Suppose $f$ is backward contracting. Then, for every integer $q \geq 0$ and every $\mathbf{n}$ in $\mathcal{S}(q)$ we have

$$
\sum_{z \in Z_{\mathbf{n}}(q)}\left|D f^{|\mathbf{n}|}(z)\right|^{-t} \leq 2^{t / d} d^{k(\mathbf{n})}\left(\tau^{p_{\mathbf{n}}(q)-q}\right)^{t / d} .
$$

Proof By definition, for each $z$ in $Z_{\mathbf{n}}(q)$ the map $f^{|\mathbf{n}|}$ maps a neighborhood $U$ of $z$ conformally onto $V_{q+1}$. Noting that $U$ is contained in $V_{p_{\mathbf{n}}(q)+1}$, and hence in $\widetilde{B}\left(\tau^{p_{\mathbf{n}}(q)+1} \delta_{0}\right)$, by Schwarz' lemma we have

$$
\left|D f^{|\mathbf{n}|}(z)\right| \geq \frac{\left(\tau^{q+1} \delta_{0} / 2\right)^{1 / d}}{\left(\tau^{p_{\mathbf{n}}(q)+1} \delta_{0}\right)^{1 / d}} \geq \frac{1}{2^{1 / d}} \tau^{-\left(p_{\mathbf{n}}(q)-q\right) / d} .
$$

Since $Z_{\mathbf{n}}(q) \leq d^{k(\mathbf{n})}$, the desired conclusion follows. 
In view of Lemma 7, Proposition 2 is a direct consequence of the following lemma.

Lemma 8 Suppose $f$ is backward contracting. Then for every sufficiently large $q \geq 0$ we have

$$
\sum_{\mathbf{n} \in \mathcal{S}(q)} d^{k(\mathbf{n})} \tau^{t \cdot p_{\mathbf{n}}(q) / d} \leq 2^{1+t / d} d\left(1-2^{-t / d}\right)^{-1}\left(R\left(\tau^{q} \delta_{0}\right)^{-1} \tau^{q-2}\right)^{t / d} .
$$

Proof Put $C_{*}:=d\left(1-2^{-t / d}\right)^{-1} 2^{t / d}$ and let $q_{0} \geq 0$ be sufficiently large so that for every $q \geq q_{0}$ we have

$$
R\left(\tau^{q} \delta_{0}\right)>\tau^{-2}\left(2 C_{*}\right)^{d / t}
$$

For each pair of integers $q \geq 0$ and $m \geq 1$, put

$$
\Xi_{t}(q, m):=\sum_{\substack{\mathbf{n} \in \mathcal{S}(q) \\|\mathbf{n}| \leq m}} d^{k(\mathbf{n})} \tau^{t \cdot p_{\mathbf{n}}(q) / d},
$$

and $\Xi_{t}(q, 0):=0$. To prove the lemma, it is enough to show that for every pair of integers $q \geq q_{0}$ and $m \geq 0$ we have

$$
\Xi_{t}(q, m) \leq 2 C_{*}\left(R\left(\tau^{q} \delta_{0}\right)^{-1} \tau^{q-2}\right)^{t / d} .
$$

We proceed by induction in $m$. By definition, for every $q$ we have $\Xi_{t}(q, 0)=0$, so inequality (6) holds trivially when $m=0$. Let $m \geq 1$ be an integer and suppose that for every $q \geq q_{0}$ the inequality (6) holds with $m$ replaced by $m-1$. Let $q \geq q_{0}$ be given and note that for every $k \geq 2$, and every $\mathbf{n}=\left(n_{1}, n_{2}, \ldots, n_{k}\right)$ in $\mathcal{S}(q)$, we have

$$
\left(n_{2}, \ldots, n_{k}\right) \in \mathcal{S}\left(p_{n_{1}}(q)\right) \text { and } p_{\left(n_{2}, \ldots, n_{k}\right)}\left(p_{n_{1}}(q)\right)=p_{\left(n_{1}, \ldots, n_{k}\right)}(q) \text {. }
$$

Conversely, for every integer $n$ in $\mathcal{N}(q)$, and every $k^{\prime} \geq 1$ and $\left(n_{1}^{\prime}, \ldots, n_{k^{\prime}}^{\prime}\right)$ in $\mathcal{S}\left(p_{n}(q)\right)$, we have

$$
\left(n, n_{1}^{\prime}, \ldots, n_{k^{\prime}}^{\prime}\right) \in \mathcal{S}(q) \quad \text { and } \quad p_{\left(n, n_{1}^{\prime}, \ldots, n_{k^{\prime}}^{\prime}\right)}(q)=p_{\left(n_{1}^{\prime}, \ldots, n_{k^{\prime}}^{\prime}\right)}\left(p_{n}(q)\right)
$$

Thus,

$$
\begin{aligned}
\Xi_{t}(q, m) & =\sum_{\substack{n \in \mathcal{N}(q) \\
n \leq m}} d\left(\tau^{t \cdot p_{n}(q) / d}+\Xi_{t}\left(p_{n}(q), m-n\right)\right) \\
& \leq \sum_{\substack{n \in \mathcal{N}(q) \\
n \leq m}} d \tau^{t \cdot p_{n}(q) / d}+d \sum_{\substack{n \in \mathcal{N}(q) \\
n \leq m-1}} \Xi_{t}\left(p_{n}(q), m-1\right) .
\end{aligned}
$$

Together with (4), and the induction hypothesis, this implies

$$
\Xi_{t}(q, m) \leq C_{*}\left(R\left(\tau^{q} \delta_{0}\right)^{-1} \tau^{q-2}\right)^{t / d}+d \sum_{\substack{n \in \mathcal{N}(q) \\ n \leq m-1}} \Xi_{t}\left(p_{n}(q), m-1\right)
$$




$$
\leq C_{*}\left[\left(R\left(\tau^{q} \delta_{0}\right)^{-1} \tau^{q-2}\right)^{t / d}+2 d \sum_{\substack{n \in \mathcal{N}(q) \\ n \leq m-1}}\left(R\left(\tau^{p_{n}(q)} \delta_{0}\right)^{-1} \tau^{p_{n}(q)-2}\right)^{t / d}\right] .
$$

Using (5), and then (4) again, we obtain

$$
\begin{aligned}
\Xi_{t}(q, m) & \leq C_{*}\left[\left(R\left(\tau^{q} \delta_{0}\right)^{-1} \tau^{q-2}\right)^{t / d}+\left(1-2^{-t / d}\right) 2^{-t / d} \sum_{\substack{n \in \mathcal{N}(q) \\
n \leq m}} \tau^{t \cdot p_{n}(q) / d}\right] \\
& \leq 2 C_{*}\left(R\left(\tau^{q} \delta_{0}\right)^{-1} \tau^{q-2}\right)^{t / d} .
\end{aligned}
$$

This completes the proof of the induction step and of the lemma.

\subsection{Proof of Theorem B}

The proof of Theorem B is at the end of this section, after a couple of lemmas.

Assume $f$ is backward contracting, fix $t>\operatorname{HD}_{\text {hyp }}(J(f))$, and consider the notation introduced in Sect. 5.1 for this choice of $t$. Put $\mathcal{O}^{-}(0):=\bigcup_{m=1}^{\infty} f^{-m}(0)$, and for each $z$ in this set denote by $m(z) \geq 1$ the unique integer $m \geq 1$ such that $f^{m}(z)=0$. Note that for every $z$ in $\mathcal{O}^{-}(0)$ there is an integer $q \geq 0$ such that the pull-back of $V_{q}$ by $f^{m(z)}$ containing $z$ is conformal. Denote by $q(z)$ the least integer $q \geq 0$ with this property.

Lemma 9 There is a constant $C_{\&}>0$ such that for every $z$ in $\mathcal{O}^{-}(0)$ satisfying $q:=q(z) \geq 1$, there exists $\mathbf{n}$ in $\mathcal{S}(q-1)$ such that the following hold:

- $m(z) \geq|\mathbf{n}|$, and $\zeta(z):=f^{m(z)-|\mathbf{n}|}(z)$ is in $Z_{\mathbf{n}}(q-1)$, and hence in $V_{p_{\mathbf{n}}(q-1)+1}$;

- $f^{m(z)-|\mathbf{n}|}$ maps a neighborhood $U(z)$ of $z$ conformally onto $V_{p_{\mathbf{n}}(q-1)}$;

- Denoting by $\zeta^{\prime}(z)$ the unique point in $U(z)$ such that $f^{m(z)-|\mathbf{n}|}\left(\zeta^{\prime}(z)\right)=0$, we have

$$
\left|D f^{m(z)}(z)\right| \geq C_{\&}\left|D f^{|\mathbf{n}|}(\zeta(z))\right|\left|D f^{m(z)-|\mathbf{n}|}\left(\zeta^{\prime}(z)\right)\right| .
$$

Proof The third assertion follows from the first and the second, together with Koebe distortion theorem. To prove the first and second assertions, we proceed by induction in $m(z)$. Let $z$ be a point in $\mathcal{O}^{-}(0)$ such that $q(z) \geq 1$ and $m(z)=1$. Then 1 is in $\mathcal{N}(q-1)$, and the desired assertions are easily seen to be satisfied with $\mathbf{n}=(1)$. Let $m \geq 2$ be an integer and suppose the desired assertions are satisfied for every $z$ in $\mathcal{O}^{-}(0)$ such that $q(z) \geq 1$ and $m(z) \leq m-1$. Let $z$ be a point in $\mathcal{O}^{-}(0)$ such that $q:=q(z) \geq 1$ and $m(z)=m$. Note that $f(z)$ is in $\mathcal{O}^{-}(0)$ and $m(f(z))=m(z)-1$. If $q(f(z)) \leq q-1$, then the pull-back of $V_{q-1}$ by $f^{m(z)-1}$ containing $f(z)$ is conformal. This implies that $m(z)$ is in $\mathcal{N}(q-1)$, and then the desired assertions are verified with $\mathbf{n}=(m(z))$. If $q(f(z)) \geq q$, then we can apply the induction hypothesis with $z$ replaced by $f(z)$; let $\mathbf{n}^{\prime}=\left(n_{1}^{\prime}, \ldots, n_{k^{\prime}}^{\prime}\right)$ be the corresponding element of $\mathcal{S}(q-1)$. If the pull-back of $U(f(z))$ by $f$ containing $z$ is conformal, then the desired assertions are verified with $\mathbf{n}=\mathbf{n}^{\prime}$. Otherwise, $n^{\prime}:=m(z)-\left|\mathbf{n}^{\prime}\right|$ is in $\mathcal{N}\left(p_{\mathbf{n}^{\prime}}(q-1)\right)$, and then the desired assertions are verified with $\mathbf{n}=\left(n_{1}^{\prime}, \ldots, n_{k^{\prime}}^{\prime}, n^{\prime}\right)$. 
Lemma 10 Assume that $f$ is backward contracting. Then for each $q \geq 0$ and $t>$ $\operatorname{HD}_{\text {hyp }}(J(f))$, we have

$$
\sum_{\substack{z \in \mathcal{O}^{-}(0) \\ q(z) \leq q}}\left|D f^{m(z)}(z)\right|^{-t}<\infty .
$$

Proof Let $\mu$ denote a conformal measure of $f$ of exponent $h:=\operatorname{HD}_{\text {hyp }}(f)$. For each $z$ in $\mathcal{O}^{-}(0)$, let $B(z)$ denote the pull-back of $V_{q+1}$ by $f^{m(z)}$ that contains $z$. By Koebe distortion theorem, there is a constant $C>1$ such that for every $z$ in $\mathcal{O}^{-}(0)$ satisfying $q(z) \leq q$, the distortion of $f^{m(z)}$ on $B(z)$ is bounded by $C$. Then for every such $z$ we have $\left|D f^{m(z)}(z)\right| \geq C^{-1} \operatorname{diam}\left(V_{q+1}\right) / \operatorname{diam}(B(z))$, and by conformality of $\mu$, we also have

$$
\mu(B(z)) \geq C^{-h}\left|D f^{m(z)}(z)\right|^{-h} \mu\left(V_{q+1}\right) .
$$

Since for a fixed integer $m \geq 1$ the sets $(B(z))_{z \in f^{-m}(0)}$ are pairwise disjoint, letting $\widetilde{C}:=C^{t} \operatorname{diam}\left(V_{q+1}\right)^{-(t-h)} \mu\left(V_{q+1}\right)^{-1}$ we have

$$
\begin{aligned}
\sum_{\substack{z \in f^{-m}(0) \\
q(z) \leq q}}\left|D f^{m}(z)\right|^{-t} & \leq \widetilde{C} \sum_{\substack{z \in f^{-m}(0) \\
q(z) \leq q}} \operatorname{diam}(B(z))^{t-h} \mu(B(z)) \\
& \leq \widetilde{C} \max _{z \in f^{-m}(0)} \operatorname{diam}(B(z))^{t-h}
\end{aligned}
$$

By [24, Theorem A], for every $\beta>0$ the sequence $\left(\max _{z \in f^{-m}(0)} \operatorname{diam}(B(z))\right)_{m=1}^{\infty}$ decreases faster than the sequence $\left(m^{-\beta}\right)_{m=1}^{\infty}$. The proposition follows summing over $m \geq 1$.

Proof of Theorem B One of the implications of the theorem is given by Proposition 1 . To prove the reverse implication, fix $t>\operatorname{HD}_{\text {hyp }}(f)$ such that

$$
\sum_{n=1}^{\infty}\left|D f^{n}(c)\right|^{-t / d}<+\infty
$$

By part 2 of Theorem 1 we have

$$
\sum_{q=0}^{\infty} R\left(\tau^{q} \delta_{0}\right)^{-t / d}<+\infty
$$

Thus, if we denote by $C_{\#}$ and $C_{\&}$ the constants given by Proposition 2 and Lemma 9 , respectively, then there is $Q \geq 1$ so that

$$
C_{\#} C_{\&}^{-t} \sum_{q=Q+1}^{\infty} R\left(\tau^{q-1} \delta_{0}\right)^{-t / d} \leq \frac{1}{2} .
$$


Taking $Q$ larger if necessary, assume that for each $z$ in $f^{-1}(0)$ we have $Q \geq q(z)$. For every pair of integers $q \geq 0$ and $m \geq 1$, put

$$
\mathcal{P}_{t}(q, m):=\sum_{\substack{z \in \mathcal{O}^{-}(0) \\ q(z)=q, m(z) \leq m}}\left|D f^{m(z)}(z)\right|^{-t},
$$

and note that by Lemma 10 we have

$$
C^{\prime}:=1+\sum_{q=0}^{Q} \sup \left\{\mathcal{P}_{t}(q, m): m \geq 1\right\}<\infty
$$

To prove that $\mathcal{P}(0, t)$ is finite, it is enough to prove that for every integer $m$ we have

$$
1+\sum_{q=0}^{+\infty} \mathcal{P}_{t}(q, m) \leq 2 C^{\prime}
$$

We proceed by induction. By our choice of $Q$, for each $z$ in $f^{-1}(0)$ we have $q(z) \leq$ $Q$. So when $m=1$ inequality (7) follows from our definition of $C^{\prime}$. Let $m \geq 2$ be an integer and suppose (7) holds with $m$ replaced by $m-1$. In view of Lemma 9, for each $q \geq Q+1$ we have

$$
\begin{aligned}
\mathcal{P}_{t}(q, m) \leq & C_{\&}^{-t}\left(\sum_{\mathbf{n} \in \mathcal{S}(q-1)} \sum_{\zeta \in Z_{\mathbf{n}}(q-1)}\left|D f^{m(\zeta)}(\zeta)\right|^{-t}\right) \\
& \cdot\left(1+\sum_{\substack{\zeta^{\prime} \in \mathcal{O}^{-}(0) \\
m\left(\zeta^{\prime}\right) \leq m-1}}\left|D f^{m\left(\zeta^{\prime}\right)}\left(\zeta^{\prime}\right)\right|^{-t}\right) .
\end{aligned}
$$

So by Proposition 2 we have

$$
\mathcal{P}_{t}(q, m) \leq C_{\#} C_{\&}^{-t} R\left(\tau^{q-1} \delta_{0}\right)^{-t / d}\left(1+\sum_{p=0}^{\infty} \mathcal{P}_{t}(p, m-1)\right) .
$$

Summing over $q \geq Q+1$, we obtain by the induction hypothesis

$$
\sum_{q=Q+1}^{\infty} \mathcal{P}_{t}(q, m) \leq \frac{1}{2}\left(1+\sum_{p=0}^{\infty} \mathcal{P}_{t}(p, m-1)\right) \leq C^{\prime}
$$

Using the definition of $C^{\prime}$, this implies (7). This completes the proof of the induction step and of the theorem.

\section{References}

1. Carleson, L., Gamelin, T.W.: Complex Dynamics. Universitext: Tracts in Mathematics. Springer, New York (1993) 
2. Denker, M., Urbański, M.: On Sullivan's conformal measures for rational maps of the Riemann sphere. Nonlinearity 4(2), 365-384 (1991)

3. Graczyk, J., Smirnov, S.: Non-uniform hyperbolicity in complex dynamics. Invent. Math. 175(2), 335-415 (2009)

4. Hubbard, J.H.: Local connectivity of Julia sets and bifurcation loci: three theorems of J.-C. Yoccoz. In: Topological Methods in Modern Mathematics, Stony Brook, NY, 1991, pp. 467-511. Publish or Perish, Houston (1993)

5. Kahn, J., Lyubich, M.: A priori bounds for some infinitely renormalizable quadratics. II. Decorations. Ann. Sci. Éc. Norm. Super. (4) 41(1), 57-84 (2008)

6. Kahn, J., Lyubich, M.: Local connectivity of Julia sets for unicritical polynomials. Ann. Math. (2) 170(1), 413-426 (2009)

7. Kozlovski, O., van Strien, S.: Local connectivity and quasi-conformal rigidity of non-renormalizable polynomials. Proc. Lond. Math. Soc. (3) 99(2), 275-296 (2009)

8. Li, H.B., Shen, W.X.: On non-uniform hyperbolicity assumptions in one-dimensional dynamics. Sci. China Math. 53(7), 1663-1677 (2010)

9. Li, H., Shen, W.: Topological invariance of a strong summability condition in one-dimensional dynamics. Int. Math. Res. Not. (2011). doi:10.1093/imrn/rns105

10. Levin, G., van Strien, S.: Local connectivity of the Julia set of real polynomials. Ann. Math. (2) 147(3), 471-541 (1998)

11. Levin, G., van Strien, S.: Bounds for maps of an interval with one critical point of inflection type. II. Invent. Math. 141(2), 399-465 (2000)

12. Lyubich, M.: Dynamics of quadratic polynomials, I-II. Acta Math. 178(2), 185-297 (1997)

13. McMullen, C.T.: Complex Dynamics and Renormalization. Annals of Mathematics Studies., vol. 135. Princeton University Press, Princeton (1994)

14. McMullen, C.T.: Hausdorff dimension and conformal dynamics. II. Geometrically finite rational maps. Comment. Math. Helv. 75(4), 535-593 (2000)

15. Milnor, J.: Dynamics in One Complex Variable, 3rd edn. Annals of Mathematics Studies, vol. 160. Princeton University Press, Princeton (2006)

16. Prado, E.A.: Ergodicity of conformal measures for unimodal polynomials. Conform. Geom. Dyn. 2, 29-44 (1998)

17. Przytycki, F., Rivera-Letelier, J.: Statistical properties of topological Collet-Eckmann maps. Ann. Sci. Éc. Norm. Super. (4) 40(1), 135-178 (2007)

18. Przytycki, F., Rivera-Letelier, J., Smirnov, S.: Equivalence and topological invariance of conditions for non-uniform hyperbolicity in the iteration of rational maps. Invent. Math. 151(1), 29-63 (2003)

19. Przytycki, F., Rivera-Letelier, J., Smirnov, S.: Equality of pressures for rational functions. Ergod. Theory Dyn. Syst. 24(3), 891-914 (2004)

20. Przytycki, F.: Lyapunov characteristic exponents are nonnegative. Proc. Am. Math. Soc. 119(1), 309317 (1993)

21. Przytycki, F.: Conical limit set and Poincaré exponent for iterations of rational functions. Trans. Am. Math. Soc. 351(5), 2081-2099 (1999)

22. Przytycki, F., Urbański, M.: Conformal Fractals: Ergodic Theory Methods. London Mathematical Society Lecture Note Series, vol. 371. Cambridge University Press, Cambridge (2010)

23. Rivera-Letelier, J.: A connecting lemma for rational maps satisfying a no-growth condition. Ergod. Theory Dyn. Syst. 27(2), 595-636 (2007)

24. Rivera-Letelier, J., Shen, W.: Statistical properties of one-dimensional maps under weak hyperbolicity assumptions (2010). arXiv:1004.0230v1

25. Sullivan, D.: Conformal dynamical systems. In: Geometric Dynamics, Rio de Janeiro, 1981. Lecture Notes in Math., vol. 1007, pp. 725-752. Springer, Berlin (1983)

26. Urbański, M.: Measures and dimensions in conformal dynamics. Bull. Am. Math. Soc. (N. S.) 40(3), 281-321 (2003) (electronic) 